\title{
UM ESTUDO SOBRE AS POSSIBILIDADES PEDAGÓGICAS DE UTILIZAÇÃO DO WHATSAPP
}

\author{
Naira Kaieski - FACCAT - naira.kaieski@gmail.com \\ Jacques Andre Grings - PPG/FACCAT - jacques.grings@ gmail.com \\ Shirlei Alexandra Fetter - PPG/FACCAT - fettershirlei@ gmail.com
}

\begin{abstract}
Resumo: O presente artigo trata sobre as possibilidades pedagógicas de utilização da ferramenta de comunicação instantânea WhatsApp como um meio facilitador no complexo processo de ensino e aprendizagem suportado por tecnologias da informação e comunicação. O trabalho traz como principais contribuições científicas um estudo aprofundado das iniciativas que já visam a utilizar o WhatsApp nas atividades pedagógicas e os resultados de uma pesquisa-ação sobre o seu uso em diferentes áreas e níveis de conhecimento. Os resultados obtidos com a pesquisa se mostram alinhados com a literatura pesquisada sobre o tema, e o uso do WhatsApp promoveu um maior engajamento, participação e colaboração dos discentes no processo de ensino e aprendizagem significativo para além dos limites físicos da sala de aula.
\end{abstract}

Palavras-chave: Mídias na Educação; WhatsApp; Tecnologias de Informação e Comunicação.

\section{A STUDY ABOUT THE PEDAGOGICAL POSSIBILITIES OF WHATSAPP UTILIZATION}

Abstract: The present article is about the pedagogical possibilities of the utilization of Mobile Instant Messaging "WhatsApp" as a facilitator in the complex teaching and learning process, supported by communication and information technologies. The main scientific contribution of this paper is based on a thoroughly study about the initiatives on using WhatsApp in pedagogical activities, and the outcomes of a research about the utilization of this tool in different areas and levels of knowledge. The results of this research match the available literature in this area; WhatsApp usage promoted a greater engagement, participation and collaboration from students in the teaching and learning process, both inside and outside the classroom.

Key words: Media in Education; WhatsApp; Communication and Information Technologies.

\section{INTRODUÇÃO}

Vivemos em uma era de modernidade líquida onde as formas estão em constante adaptação ao contexto. Bauman (2000) afirma que, na modernidade líquida, os sujeitos estão em fluxo contínuo, são voláteis, flexíveis e instáveis. As linguagens consideradas temporais (verbo, som e vídeo) especializam-se nas cartografias líquidas e invisíveis do ciberespaço, enquanto as linguagens espaciais (imagens, diagramas e fotos) fluidificamse nas enxurradas e circunvoluções dos fluxos. É nesse contexto que se desenvolve o ensino híbrido, que é uma abordagem pedagógica que combina atividades presenciais e atividades realizadas por meio das tecnologias digitais de informação e comunicação (VALENTE, 2015). De certa forma, a essência do ensino híbrido já vem sendo aplicada mesmo sem a conceitualização de sua existência. Nessa estratégia pedagógica, o foco do processo de aprendizagem é o aluno, e o conteúdo e as instruções sobre um determinado tema curricular são disponibilizados através de diferentes ambientes tecnológicos.

As Tecnologias da Informação e Comunicação (TICs) podem ser utilizadas como uma estratégia para aproximar os estudantes das atividades e conteúdos escolares, 
principalmente se consideradas as grandes mudanças que vêm ocorrendo nos processos de ensino e aprendizagem motivadas justamente pelo desenvolvimento de novas ferramentas e tecnologias (MATTAR, 2014). A adoção de plataformas e ferramentas digitais pode contribuir significativamente para que cada aluno desenvolva habilidades e competências compatíveis com as novas demandas sociais, construindo um percurso próprio de aprendizagem a partir das suas necessidades. Dessa forma, os discentes são capazes de construir experiências de aprendizagem coletivas e colaborativas, potencialmente reformulando espaços e tempos escolares e ampliando o papel do professor como mediador de conhecimento (LIMA; ROSENDO, 2014). Tarouco (2013) afirma que a preocupação com o uso criativo e inovador das TICs deve estar presente nas pesquisas relacionadas com as habilidades dos estudantes para atuar produtivamente na sociedade digital. Os recursos e serviços atualmente disponíveis facilitam o engajamento do discente em tarefas nas quais se envolve de forma ativa, construtivista, intencional, autêntica e cooperativa.

Com a nova geração de discentes, considerados nativos digitais, um novo formato de ensino, adequado às demandas dos discentes, se faz necessário. Um dos pilares dessa nova geração é a utilização fluente das TICs, sendo, portanto, atribuída aos docentes a incumbência de atualização de suas práticas pedagógicas para que possam acompanhar o desenvolvimento das novas mídias de forma que consigam promover a inserção dessas novas ferramentas digitais em suas atividades e, ao mesmo tempo, acompanhar a evolução dos alunos. O docente possui uma sala de aula ampliada para trabalhar, que se mescla, hibridiza constantemente e onde a tecnologia proporciona a integração de todos os espaços e tempos. O ensinar e o aprender acontecem em uma interligação simbiótica, profunda e constante entre os mundos físico e digital (MORAN, 2015).

Tão logo uma nova tecnologia ou ferramenta é disponibilizada no universo digital, surgem pesquisas que visam a explorar sua potencial utilização nas diversas áreas da sociedade, e uma delas é a educação. Um bom exemplo foram os ensaios produzidos sobre a utilização das redes sociais na educação através da adoção do Facebook quando este se tornou uma ferramenta de rede social amplamente popular. Este artigo objetiva estudar a potencial utilização do WhatsApp nas atividades pedagógicas, explorando os projetos já realizados e a aplicação de uma pesquisa-ação que visou a analisar a inserção desta ferramenta no processo de ensino e aprendizagem. O WhatsApp é um aplicativo para dispositivos móveis, o que possibilita uma forma de comunicação instantânea. Mattar (2014) define o WhatsApp como uma ferramenta de comunicação rápida e promissora a ser utilizada como uma plataforma de apoio à educação, visto que possibilita o envio de textos, imagens, sons e vídeos e a criação de grupos de usuários. Já Moran (2015) cita outro aspecto positivo em relação à forma de comunicação proporcionada por esta ferramenta, que é a utilização de uma linguagem mais familiar, maior espontaneidade e fluência constante de imagens, ideias e vídeos.

\section{TECNOLOGIA NA EDUCAÇÃO}

Ensinar com novas tecnologias é um grande desafio e, de acordo com o autor Perrenoud (2000), a escola não pode ignorar o que se passa no mundo, já que as novas Tecnologias da Informação e Comunicação transformam espetacularmente não só as maneiras de comunicar, mas também de estudar, de trabalhar, de decidir e de pensar. Com esse pensamento é imprescindível que os docentes busquem atualização e aprimoramento constantes para atender um contingente de discentes mais críticos e fluentes na utilização dos recursos digitais. Segundo Moran (2013), as tecnologias móveis que chegam às mãos de alunos e professores trazem enormes desafios de como organizar os processos de aprendizagem de forma interessante, atraente e eficiente 
dentro e fora da sala de aula, aproveitando o melhor de cada ambiente, o presencial e o digital. Perrenoud (2000) explica que os professores que sabem o que as novidades tecnológicas aportam, bem como seus perigos e limites, podem decidir, com conhecimento de causa, dar-lhes um amplo espaço em sua classe ou utilizá-las de modo bastante marginal. Contudo, de acordo com Moran (2013), muitos professores sentem cada vez mais claro o descompasso no domínio das tecnologias e evitam a sua utilização com receio de revelar seu desconhecimento para os alunos.

O desenvolvimento da Web 2.0, dos dispositivos móveis e inteligentes, da internet e das coisas e a popularização do acesso à internet com banda larga propulsionam as novas tecnologias na direção da integração, da instantaneidade, da comunicação audiovisual e interativa. Na educação, emerge o termo aprendizagem móvel, que, segundo o relatório "Diretrizes de políticas para a aprendizagem móvel", divulgado pela UNESCO em 2014, envolve o uso de tecnologias móveis, isoladamente ou em combinação com outras tecnologias de informação e comunicação, a fim de permitir a aprendizagem a qualquer hora e em qualquer lugar. Conforme Moran (2013), há um campo enorme de possibilidades de comunicação entre as pessoas, grupos pequenos e grandes na educação e na vida.

Assim como já ocorreu com outras ferramentas, como a da rede social Facebook, o estudo sobre o emprego do aplicativo WhatsApp na educação como ferramenta de comunicação instantânea pode trazer resultados benéficos ao processo de ensino e aprendizagem alinhado às perspectivas dos discentes nativos digitais. Segundo a UNESCO (2014), as mensagens enviadas através de aparelhos móveis são mais rápidas, confiáveis e eficientes do que as enviadas por canais alternativos de comunicação. A pesquisa TIC Kids Online Brasil 2013, do Comitê Gestor da Internet no Brasil (2014), constatou que as atividades realizadas na internet com maior frequência por crianças e adolescentes com idade entre 11 e 17 anos são aquelas primariamente relacionadas a formas de comunicação e interação social na rede, conforme apresentado na Figura 1. Um número elevado de crianças e adolescentes (74\%) declarou utilizar todos os dias ou quase todos os dias mensagens instantâneas como o WhatsApp para se comunicar.

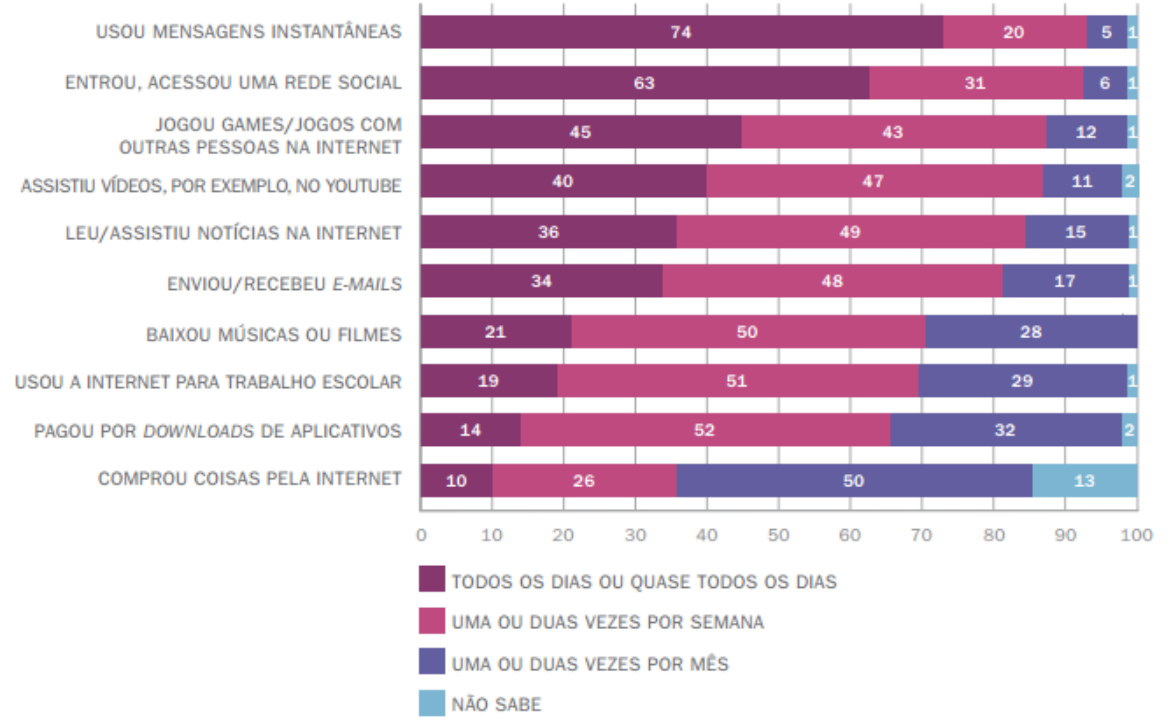

Figura 1 - Proporção de crianças/adolescentes por frequência de uso da internet para cada atividade realizada no último mês (percentual sobre o total de usuários de internet de 11 a 17 anos).

Fonte: Pesquisa sobre o uso da internet por crianças e adolescentes no Brasil - TIC Kids Online Brasil 2013 (p. 122). 


\subsection{WhatsApp}

Logo que uma nova ferramenta ou tecnologia é desenvolvida e disponibilizada, surgem pesquisadores interessados em explorar a sua utilização em diversas áreas. $\mathrm{O}$ WhatsApp Messenger é um aplicativo de mensagens multiplataforma lançado em 2009, que rapidamente se popularizou principalmente nos últimos anos. Segundo Smith (2015), o WhatsApp totalizou 800 milhões de usuários, em 2015, com uma média de 1 milhão de novos usuários por dia, chegando a um volume de 30 bilhões de mensagens enviadas diariamente. A Figura 2 mostra o espetacular crescimento no número de usuários ativos do WhatsApp nos últimos anos.

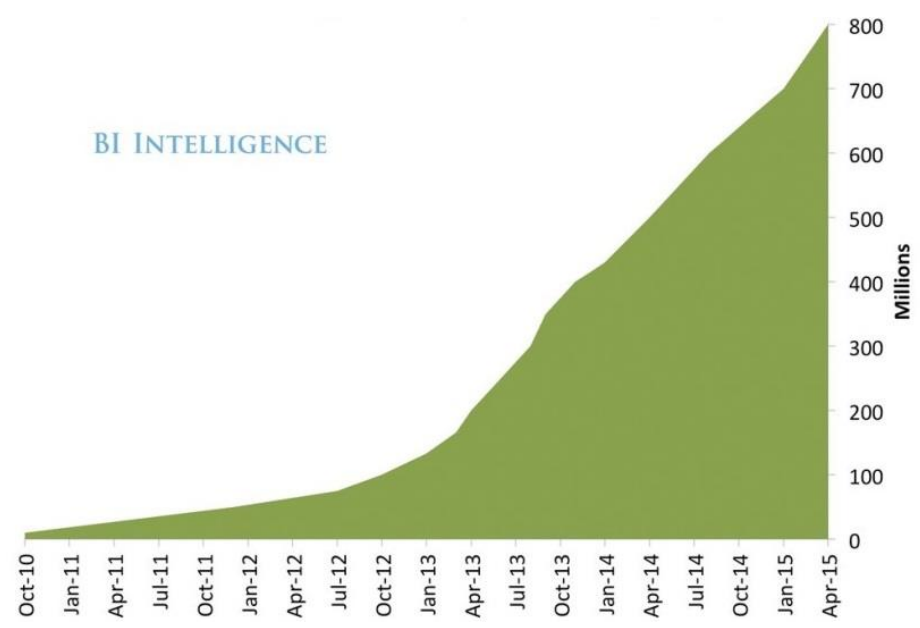

Figura 2 - Estatística global da evolução mensal do número de usuários do WhatsApp ativos de outubro de 2010 a janeiro de 2015.

Fonte: KIM, 2015

Inicialmente, o WhatsApp era uma ferramenta de comunicação instantânea apenas para dispositivos móveis, mas as versões mais recentes podem ser utilizadas tanto em dispositivos móveis (smartphones e tablets) como em computadores pessoais através dos navegadores de internet Google Chrome, Mozilla Firefox e Opera. O aplicativo disponibiliza diversos recursos interessantes de comunicação como o envio de texto, fotos, áudios, vídeos e recentemente passou a disponibilizar a opção de efetuar ligações (WHATSAPP, 2015). Outra funcionalidade que pode ser particularmente importante para as atividades pedagógicas é a confirmação do recebimento e da leitura das mensagens enviadas.

Uma das grandes vantagens financeiras do WhatsApp é o seu custo, pois o envio das mensagens é gratuito, sendo necessária apenas uma conexão com a internet para viabilizar o envio de mensagens e a realização de ligações. Assim o usuário pode fazer uso de uma conexão com a internet já existente no ambiente onde ele se encontra, como escolas, comércio e locais públicos.

\section{PESQUISAS RELACIONADAS}

Atualmente, as tecnologias móveis estão verdadeiramente prontas para transformar o aprendizado dentro e fora da sala de aula. É chegado o momento de as instituições educacionais, em todos os níveis, desenvolverem programas de ensino e aprendizagem voltados para o uso das tecnologias móveis, objetivando colher os benefícios da redução de custos aliada a um ensino mais eficaz, com maior aproveitamento do aluno (CENTRE FOR DIGITAL EDUCATION, 2011). Como já foi apresentado, o WhatsApp é uma ferramenta poderosa de comunicação que vem atraindo um número 
espetacular de novos usuários no mundo. Da mesma forma, cresce o número de pesquisas que visam a explorar as possibilidades de uso do WhatsApp na educação. Este capítulo traz algumas dessas inciativas e os resultados obtidos.

Um dos benefícios de empregar a tecnologia na educação é expandir para além do espaço físico do educandário as possibilidades de aprendizagem. Com esse objetivo, uma escola da cidade de Riyadh, na Arábia Saudita, desenvolveu um projeto para utilizar o WhatsApp como uma ferramenta de apoio para que os discentes pudessem continuar aprendendo também fora da sala de aula. Foram criados grupos no WhatsApp onde os discentes e docentes discutiam sobre as lições e tiravam dúvidas. Para estimular o aprendizado, os professores enviavam perguntas ao grupo e os estudantes com as melhores respostas recebiam bonificações semanais. Um dos docentes dessa instituição adotou como estratégia enviar a pergunta ao grupo logo no início da manhã, e assim os alunos chegavam à aula já motivados (ARAB NEWS, 2015).

Rambe e Bere (2013) conduziram um estudo na África do Sul com o objetivo de investigar os potenciais pedagógicos do emprego do envio de mensagens eletrônicas através do WhatsApp para criar espaços dialógicos alternativos propensos ao engajamento e à colaboração entre estudantes em um ambiente informal. Os resultados obtidos sugerem uma elevada participação dos estudantes por meio das comunidades para compartilhamento de conhecimento e progressiva adaptação das práticas pedagógicas dos docentes que não utilizavam os recursos tecnológicos por não possuírem a fluência necessária para integrar as TICs às suas práticas tradicionais. A forma de comunicação proporcionada pelo WhatsApp gerou impactos positivos na participação dos alunos, promovendo a aprendizagem socioconstrutivista através de discussões espontâneas, aumentando a autoconfiança do estudante para se envolver nas discussões e melhorando o compartilhamento do conhecimento e dos recursos gerados coletivamente em vários espaços.

Plana (2015) desenvolveu um projeto na Espanha que visou a explorar as vantagens e desvantagens da utilização doWhatsApp para melhorar as habilidades de leitura dos alunos durante a aprendizagem da língua inglesa. O WhatsApp foi utilizado para proporcionar o envio das atividades aos discentes, que deveriam acessar o endereço enviado e responder aos questionamentos da atividade. Uma informação relevante deste projeto se refere à motivação dos participantes, que, após o encerramento do estudo, responderam a uma pesquisa que apontou que mais de $90 \%$ dos entrevistados reconheceram que a sua participação no projeto tinha aumentado a sua motivação para a leitura em inglês. Esse resultado mostra que o emprego de tecnologias mais aderentes ao cotidiano dos discentes fortalece o seu engajamento nas atividades.

A pesquisa de Mudliar e Rangaswamy (2015) realizada na Índia obteve um aspecto inesperado em relação às interações entre gênero, que são muito relevantes no âmbito educacional. Nesse país, a segregação social e de gênero é muito forte, e os meninos e as meninas são separados no ambiente escolar. Contudo, no mundo digital, por meio da troca de mensagens no WhatsApp, houve um rompimento dessas barreiras, e os alunos interagiram. A pesquisa sugere que o WhatsApp cria um ambiente de baixo risco, o que facilita novas interações com o gênero diferente, tornando a sala de aula digital livre de restrições sociais e de gênero. Curiosamente, essa interação entre gênero raramente era replicada no mundo real, mesmo entre discentes que estudaram juntos por um longo período. Corroborando os aspectos sociais apontados, o trabalho da pesquisadora Amry (2014) demonstra uma maior efetividade na interação social através do WhatsApp em relação às relações exclusivamente presenciais. Ao observar as atitudes dos estudantes, surgiram fortes indícios de que o uso do WhatsApp tornou o processo de aprendizagem mais fácil, favorecendo a resolução de problemas e o compartilhamento de informações. 
As pesquisas apresentadas elucidam principalmente aspectos positivos da utilização do WhatsApp na educação, como os apontados por Bansal e Joshi (2014), em que os estudantes consideraram o uso desta ferramenta interessante e pedagogicamente útil. Ngaleka e Uys (2013) indicam uma significativa colaboração e aprendizagem adquirida fora da sala de aula sem a influência do professor. Rambe e Chipunza (2013) acrescentam que o uso do WhatsApp promove a aprendizagem significativa livre de contexto. Os pesquisadores Bouhnik e Deshen (2014) apresentam como vantagens educacionais a criação de um ambiente agradável e de maior proximidade entre os discentes, acessibilidade aos materiais de aprendizagem e a disponibilidade dos professores em interagir além do horário das aulas.

Como esta ferramenta de comunicação é geralmente utilizada como apoio em um ambiente de ensino híbrido, não sendo obrigatória sua utilização pelos discentes, aspectos negativos não são evidenciados fortemente nos trabalhos relacionados. Contudo Bouhnik e Deshen (2014) apontam como desvantagens o fato de que nem todos os estudantes possuem dispositivos móveis, a possibilidade, em alguns casos, de os docentes acabarem enviando mensagens em demasia para os estudantes e a irreal expectativa dos discentes quanto à disponibilidade integral dos professores para tirar dúvidas a qualquer momento do dia e da noite.

\section{PROCEDIMENTOS METODOLÓGICOS}

Considerando a premissa de que a utilização da mídia WhatsApp em sala de aula pode se tornar uma ferramenta produtiva no âmbito de promover uma nova dinâmica no processo de ensino e aprendizagem, a pesquisa-ação foi escolhida para ser aplicada neste ensaio. A pesquisa buscou investigar o impacto da utilização do WhatsApp como ferramenta de apoio no processo de ensino e aprendizagem expandindo as fronteiras da sala de aula tradicional. A opinião e a impressão dos principais atores envolvidos no processo, ou seja, o discente e o docente, foram consideradas muito relevantes frente à utilização deste meio dinâmico, abrangente, instantâneo e atual de comunicação como ferramenta de apoio às atividades pedagógicas.

Durante a aplicação do projeto, houve momentos de intervenção junto ao objeto de pesquisa que visaram ao seu alinhamento aos docentes e discentes envolvidos. A intervenção do pesquisador no objeto de pesquisa caracteriza a pesquisa-ação, que consiste em uma forma de investigação-ação definida como toda tentativa continuada, sistemática e empiricamente fundamentada de aprimorar a prática. Tripp (2005) ainda afirma que a pesquisa-ação educacional é principalmente uma estratégia para o desenvolvimento de professores e pesquisadores de modo que eles possam utilizar suas pesquisas para aprimorar seu ensino e, em decorrência, o aprendizado de seus alunos.

A pesquisa foi aplicada em duas instituições de ensino da cidade de Taquara, Rio Grande do Sul, apresentadas abaixo:

- Escola de idiomas: turma com 3 alunos, idade entre 15 e 23 anos, cursando o nível intermediário de Inglês;

- Escola técnica: turma com 13 alunos, idade entre 18 e 38 anos, cursando a disciplina de Programação I do Curso Técnico em Informática;

A pesquisa compreendeu uma etapa inicial de intervenção junto aos docentes onde o WhatsApp foi apresentado, visando a estender o conhecimento da ferramenta que os docentes já possuíam. Nesta fase inicial, houve uma tentativa conjunta entre pesquisador e docente de verificar como o WhatsApp poderia ser utilizado, objetivando potencializar a apropriação de conhecimento dos discentes, considerando o contexto de cada turma e o conteúdo programático a ser desenvolvido. Foi decidido que seria criado um grupo no WhatsApp com todos os alunos da turma mais o docente e o pesquisador. 
O grupo seria usado para que os alunos pudessem postar suas dúvidas e interagir, mandando material pertinente ao conteúdo programático. Já o docente deveria interagir com o grupo, respondendo aos questionamentos e também o instigando, enviando material, notícias relevantes e questionamentos.

A segunda etapa do projeto foi a apresentação do mesmo aos discentes que compreendeu uma explicação sobre a dinâmica esperada na interação, a criação do grupo, adição dos membros do grupo e a troca de algumas mensagens para testar o ambiente. Foi acordado com os discentes que apenas temas relevantes com o objeto de estudo seriam postados no grupo.

Ambas as instituições de ensino disponibilizam acesso à internet via rede sem fio para os seus alunos, possuindo todos eles dispositivo móvel compatível com o WhatsApp. Todos os discentes afirmaram que já conheciam o WhatsApp e o utilizavam com frequência, fazendo parte de outros grupos, fato que facilitou a implantação do projeto. A última etapa do projeto foi constituída por um momento de reflexão com os discentes e os docentes sobre os resultados alcançados. Aos discentes foi solicitado que respondessem a um questionário de avaliação do projeto, composto por questões objetivas e dissertativas, para que pudessem expor a sua opinião. Contudo, embora a fase de coleta e análise de dados tenha sido concluída, a utilização do WhatsApp nas turmas onde o projeto foi aplicado irá prosseguir dados os resultados positivos obtidos.

\section{RESULTADOS ENCONTRADOS}

O projeto teve uma duração de três semanas em cada instituição, o que correspondeu a um ou mais conteúdos programáticos do currículo previsto. Em geral, os discentes tiveram uma participação ativa na troca de mensagens, mas é possível observar, também no mundo digital, que alguns alunos não se sentem seguros para postar suas dúvidas. Entretanto, à medida que o projeto era desenvolvido e o docente estimulava o grupo, foi possível observar um aumento no fluxo de mensagens e no número de discentes ativos nos grupos. Os alunos passaram a interagir com mais frequência e naturalidade, bem como a desenvolver as atividades propostas. No geral, o engajamento da turma aumentou, o que foi mensurado pelas atividades desenvolvidas. A Figura 3 apresenta uma troca de mensagens no grupo do WhatsApp referentes ao aprendizado do idioma inglês.

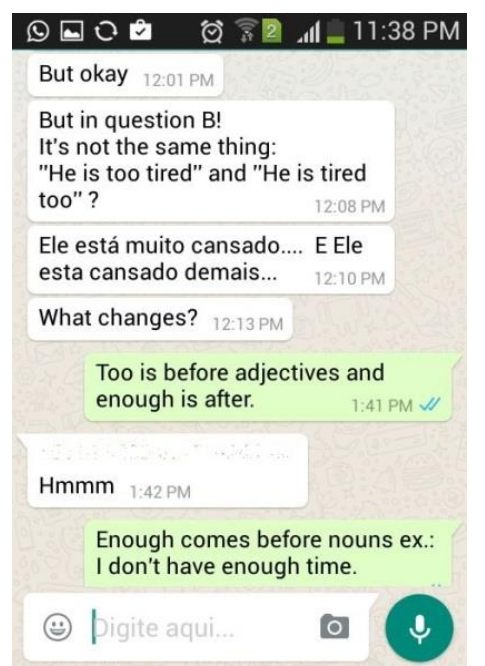

Figura 3 - Mensagens trocadas no grupo do WhatsApp sobre aprendizado de idiomas. Fonte: A autora

Ao final do projeto, não foi observada evasão de discentes, e o fluxo de mensagens teve um aumento significativo. Para a elaboração deste artigo, além da observação das 
mensagens no grupo e das conversas com os docentes, foi solicitado aos discentes que respondessem a um questionário que teve a finalidade de obter dados relacionados com a visão dos discentes no que diz respeito ao uso do WhatsApp como apoio pedagógico. As respostas dos discentes apontam que $87.5 \%$ acharam o uso do WhatsApp produtivo nas atividades pedagógicas e $25 \%$ não se sentiam totalmente à vontade para expor dúvidas através das mensagens para o grupo. Todos os docentes avaliaram a experiência como positiva, mas se mostraram preocupados com a possibilidade de um grande número de mensagens para gerenciar caso fosse criado um grupo no WhatsApp para cada turma em que lecionam.

\section{CONCLUSÃO}

O WhatsApp, embora seja uma ferramenta de comunicação amplamente conhecida em todo o mundo, ainda tem sido pouco explorada em projetos educacionais. Assim, este trabalho apresentou uma pesquisa bibliográfica sobre o estado da arte da utilização do WhatsApp em atividades pedagógicas e o resultado de uma pesquisa-ação onde o WhatsApp foi introduzido como uma ferramenta de apoio às atividades pedagógicas de duas instituições de ensino da cidade de Taquara, no Rio Grande do Sul.

A pesquisa bibliográfica enfatiza um número considerável de iniciativas que estão sendo desenvolvidas ao redor do mundo, visando a empregar o WhatsApp na educação. Em geral, os resultados obtidos foram exitosos, enfatizando o potencial desta ferramenta que está inserida no cotidiano de um número crescente de usuários. Dentre os benefícios mais significativos apontados pelas pesquisas, temos o rompimento das barreiras sociais e de gênero na comunicação entre os discentes, o baixo custo, a acessibilidade, a interatividade e a aprendizagem colaborativa e significativa além do espaço do educandário. Alguns dos aspectos negativos citados são os relacionados ao acesso à tecnologia e à adequação das práticas pedagógicas dos docentes às novas tecnologias e meios de comunicação.

O principal foco da utilização do WhatsApp na aplicação do projeto foi explorar as possibilidades de interatividade, colaboração e engajamento que a ferramenta possibilita. A literatura relacionada à motivação e à aprendizagem colaborativa mostra que o desempenho e a aprendizagem dos discentes dependem não só do interesse no assunto, mas também do relacionamento entre os alunos, do gerenciamento das diferenças individuais, dos traços de personalidade, das origens culturais, das diferenças de gênero e da sala de aula como um ambiente de aprendizagem (KIRCHNER; RAZMERITA, 2015).

O resultado da pesquisa-ação sobre a utilização do WhatsApp no contexto educacional proposto no projeto confirmou as referências encontradas na literatura. A avaliação geral tanto dos docentes quanto dos discentes foi positiva principalmente pela facilidade de resolução de dúvidas. Um dos discentes, em sua avaliação do projeto, enfatizou: "Fica mais fácil resolver as dúvidas, sempre tem alguém conectado respondendo." É preciso considerar que todos os docentes e discentes afirmaram já conhecer a ferramenta e participar de outros grupos, fato que pode ter facilitado a adesão ao projeto e o engajamento na interação e na colaboração.

\section{Referências}

AMRY, Aicha Blehch. The Impact of WhatsApp Mobile Social Learning on the Achievement and Attitudes of Female Students Compared with Face to Face Learning in the Classroom. European Scientific Journal. Volume 10, Número 22, Páginas 116-136, 2014. 
ARAB NEWS. WhatsApp comes in handy for students and teachers. Disponível em:<http://www.arabnews.com/news/540941?page=1>. Acesso em: 05 set. 2015.

BANSAL, Tulika; JOSHI, Dhananjay. A Study of Students' Experiences of Mobile Learning. Global Journal of Human-Social Science. Volume 14, Número 4, Páginas 26-33, 2014.

BAUMAN, Zygmunt. Liquid Modernity. Blackwell Publishers Inc: Malden, 2000.

BOUHNIK, Dan; DESHEN, Mor. WhatsApp Goes to School: Mobile Instant Messaging between Teachers and Students. Journal of Information Technology Education, Volume 13, Páginas 217-231, 2014.

CENTRE FOR DIGITAL EDUCATION. Mobile Learning: What it takes, how to get there. USA: Sprint, 2011.

CETIC. BR. TIC Kids Online Brasil 2013 - Pesquisa sobre o uso da Internet por crianças e adolescentes no Brasil. São Paulo: Comitê Gestor da Internet no Brasil, 2014.

KIM, Eugene. WhatsApp's growth is incredible: It just added another 100 million new users in 4 months. Disponível em: $<\mathrm{http}: / / w w w . b u s i n e s s i n s i d e r . c o m / w h a t s a p p-$ passes-800-million-monthly-active-users-2015-4>. Acesso em: 03 set. 2015.

LIMA, Ana Lúcia D’Império; ROSENDO, Rosi. Séries finais do ensino fundamental: o papel das TIC na etapa mais desafiadora do ensino básico. In:

CETIC. BR. Pesquisa sobre o uso das Tecnologias de Informação e Comunicação nas escolas brasileiras - TIC Educação 2013. São Paulo: Comitê Gestor da Internet no Brasil, 2014.

MATTAR, João. Design educacional: educação a distância na prática. 1. ed. São Paulo: Artesanato Educacional, 2014.

MORAN, José Manuel. Educação híbrida: Um conceito-chave para a educação hoje. In:

BACICH, Lilian; NETO, Adolfo Tanzi; TREVISANI, Fernando de Mello. Ensino híbrido: personalização e tecnologia na educação. Porto Alegre: Penso, 2015.

MORAN, José Manuel. A educação que desejamos - Novos desafios e como chegar lá. 5.ed. Campinas: Papirus, 2013.

MORAN, José Manuel; MASETTO, Marcos T.; BEHRENS, Marilda Aparecida. Novas tecnologias e mediação pedagógica. 21.ed. Campinas: Papirus, 2013.

MUDLIAR, Preeti; RANGASWAMY, Nimmi. Offline Strangers, Online Friends: Bridging Classroom Gender Segregation with WhatsApp. 33rd Annual ACM Conference on Human Factors in Computing Systems, Seoul, Páginas 3799-3808, 2015.

NGALEKA, Abulela; UYS, Walter. M-Learning WithWhatsApp: A Conversation Analysis. Proceedings of the 8th International Conferenceon e-Learning. South Africa, 2013.

Organização das Nações Unidas para a Educação, a Ciência e a Cultura. Diretrizes de políticas da UNESCO para a aprendizagem móvel. Brasília: UNESCO, 2014. 
PERRENOUD, Philippe. 10 Novas competências para ensinar. Convite à viagem. Porto Alegre: Artmed, 2000.

PLANA, Mar Gutiérrez et al. Improving learners' reading skills through instant short messages: A sample study using WhatsApp. 4th World CALL Conference, Glasgow, 10-13 julho, 2013.

KIRCHNER, Kathrin; RAZMERITA, Liana. Collaborative Learning in the Cloud A Cross-Cultural Perspective of Collaboration. 26th ACM Conference on Hypertext \& Social Media. Chipre, Páginas 333-336, 2015.

RAMBE, Patient; BERE, Aaron. Using mobile instant messaging to leverage learner participation and transform pedagogy at a South African University of Technology. British Journal of Educational Technology, Volume 44, Número 3, 2013.

RAMBE, Patient; CHIPUNZA, Crispen. Using mobile devices to leverage student access to collaboratively-generated resources: A case of WhatsApp instant messaging at a South African University. International Conference on Advanced Information and Communication Technology for Education. 2013.

SMITH, Craig. By the Numbers: 40 Amazing WhatsApp Statistics. Disponível em: <http://expandedramblings.com/index.php/whatsapp-statistics $>$. Acesso em: 01 set. 2015.

TAROUCO, Liane Margarida. Um panorama da fluência digital na sociedade da informação. In:

BEHAR, Patricia Alejandra. Competências em Educação a Distância. Porto Alegre: Penso Editora Ltda, 2013.

TRIPP, David. Pesquisa-ação: uma introdução metodológica. Educação e Pesquisa. São Paulo, Número 3, Volume 31, Páginas 443-466, set./dez. 2005.

VALENTE, José Armando. O ensino híbrido veio para ficar. In:

BACICH, Lilian; NETO, Adolfo Tanzi; TREVISANI, Fernando de Mello. Ensino híbrido: personalização e tecnologia na educação. Porto Alegre: Penso, 2015.

WHATSAPP. Disponível em:<http://www.whatsapp.com>. Acesso em: 01 set. 2015. 